\title{
The Efficacy and Safety of Bipolar Radiofrequency Treatment with Non-Insulated Penetrating Microneedles for Acne Vulgaris and Acne Scars
}

\author{
Ganesh S. Pai ${ }^{1}$ \\ Mouhamad Kashmar ${ }^{2}$ \\ Young Koo Kim ${ }^{3}$ \\ Jongju $\mathrm{Na}^{4}$
}

'DERMA-CARE, Skin and Cosmetology Center,
Karnataka, India
${ }^{2}$ Cosmoderm Clinics, Jeddah, Saudi Arabia,
${ }^{3}$ Yonsei Star Skin \& Laser Clinic, Seoul, Korea
${ }^{4}$ Department of Anatomy, Soonchunhyang
University College of Medicine, Cheonan, Korea

Received April 12, 2015

Accepted April 23, 2015

\footnotetext{
Correspondence

Jongju $\mathrm{Na}$

Department of Anatomy, Soonchunhyang University College of Medicine, Soonchunhyang6gil 31, Dongnam-gu, Cheonan 330-721, Korea Tel: +82-31-8017-7893

Fax: +82-31-8016-7894

E-mail: clavius1dhotmail.com

(c) Korean Society for Laser Medicine and Surgery

(c) This is an open access article distributed under the terms of the Creative Commons Attribution NonCommercial License (http://creativecommons.org/ licenses/by-nc/4.0) which permits unrestricted noncommercial use, distribution, and reproduction in any medium, provided the original work is properly cited.
}

\author{
Background and Objectives \\ Bipolar radiofrequency (RF) devices equipped with fractional RF \\ microneedles have been shown to be clinically effective and safe for the \\ treatment of moderate to severe acne vulgaris lesions and acne scars. \\ We aimed to determine the efficacy and safety of using non-insulated \\ penetrating microneedles to deliver bipolar RF energy for treating acne \\ vulgaris and acne scars.
}

\section{Materials and Methods}

Improvement in moderate to severe acne vulgaris and mild to severe acne scarring was evaluated in 316 patients (195 males and 121 females; ages ranging from 18-34 years; Fitzpatrick skin types IV-V) treated with two to four sessions of bipolar non-insulated microneedle treatment RF at onemonth intervals.

\section{Results}

At two months after the final bipolar RF treatment, clinical assessment revealed grade 1 improvement in $21(6.6 \%)$ of the 316 patients, grade 2 improvement in 78 (24.7\%), grade 3 improvement in $133(42.1 \%)$, and grade 4 improvement in 84 (26.6\%). Temporary aggravation of acne vulgaris or folliculitis, which spontaneously resolved within three weeks, was noted in 9 patients. Otherwise, no remarkable side effects were recorded.

\section{Conclusion}

We suggest that bipolar non-insulated microneedle RF treatment can be effectively and safely used to treat acne vulgaris and acne scars in patients with Fitzpatrick skin type IV-V.

\section{Key words}

Radiofrequency; Bipolar; Non-insulated; Microneedle; Acne vulgaris; Acne scar 


\section{INTRODUCTION}

Recently, technically innovative treatments for acne vulgaris and acne scars have been increasingly used to meet patient demands for greater convenience and efficacy than achievable with oral and topical medications. In the literature, the efficacy and safety of various light, laser, and radiofrequency (RF) devices, including intense pulsed light for photodynamic therapy, pulsed-dye lasers, fractional ablative carbon dioxide (CO2) lasers, non-ablative 1,450-nm diode lasers, and fractional RF using penetrating electrodes, have been reported for the treatment of acne vulgaris lesions and/or acne scars. ${ }^{1-4}$ These devices achieve their desired therapeutic effects on inflammatory acne lesions via a number of different mechanisms: pulsed-dye laser therapy seeks to reduce vascularity by being absorbed by hemoglobin and modulating inflammatory processes, as well as killing Propionibacterium acnes; ${ }^{1-4}$ non-ablative $1,450-n m$ diode lasers are used to induce thermal damage to sebaceous glands in an attempt to reduce sebum production; ${ }^{5-6}$ and sublative RF treatment strives to reduce sebaceous gland activity and promote dermal architecture remodeling via thermal stimulation. . $^{7-10}$

While the use of fractional RF devices equipped with penetrating microneedle electrodes has also been shown to be effective in the treatment of moderate and severe acne scarring, ${ }^{11}$ a predictive model of minimally invasive bipolar RF treatment has suggested that electrodes must be insulated in order to preserve the epidermis. ${ }^{12}$ However, a study of non-insulated microneedle fractional RF treatment revealed that the delivery of RF energy via non-insulated microneedles is safe and effective for the treatment of scars and photoaging. ${ }^{13}$ Accordingly, our study was designed to evaluate the effectiveness and safety of bipolar non-insulated microneedle RF treatment in 316 patients with inflammatory acne vulgaris and/or acne scar lesions from three different countries (India, Saudi Arabia, and Koreal via retrospective analysis of medical records and clinical photographs.

\section{MATERIALS AND METHODS}

\section{Patients}

We retrospectively reviewed the medical records of and clinical photographs for 316 patients $(195$ males and 121 females; mean age of 28.2; ages ranging from 1834 years; Fitzpatrick skin types IV-V) treated with bipolar non-insulated microneedle RF treatment for moderate to severe acne vulgaris and/or mild to severe acne scarring
(Table 1). Patients were excluded from this study if they had received systemic retinoid therapy, 1,450-nm diode laser treatment, 595-nm pulsed-dye laser treatment, photodynamic therapy with intense pulsed light, nonablative erbium:glass fractional laser treatment, or fractional CO2 laser treatment within the last six months. Patients were also excluded if they had been treated with systemic and topical antibiotics, intralesional corticosteroid injections, incision and drainage, or surgical excision within the previous month. Finally, patients with a high probability of becoming pregnant or a propensity for keloids or immunosuppression were excluded.

\section{Bipolar RF treatment}

Patients were treated with two to four sessions of bipolar non-insulated microneedle RF treatment (Scarlet; Viol, Kyeonggi, Korea) at one-month intervals. Prior to application of local anesthesia, the face was cleansed with a mild soap and 70\% alcohol. Topical EMLA cream leutectic mixture of $2.5 \%$ lidocaine $\mathrm{HCl}$ and $2.5 \%$ prilocaine; AstraZeneca AB, Södertälje, Sweden) was applied to the entire face under occlusion one hour before administering treatment. The treatment settings included a microneedle penetration depth of $3 \mathrm{~mm}$, an intensity of 7, and a RF conduction time of 300 msec. RF energy was emitted twice over the entire face, and an additional pass was delivered to severe pustular lesions and/or acne scars. Patients with a history of herpes virus infection were prophylactically prescribed oral valacyclovir hydrochloride (Valtrex; GlaxoSmithKline, Research Triangle Park, NC, USA) for three days. The use of a noncomedogenic moisturizer several times daily for a few days after each treatment session was recommended in order to promote wound healing and prevent dryness. Patients were instructed to avoid overexposure to sunlight and to apply a broad-spectrum sunscreen after posttherapy crusting subsided. Patients were also instructed to avoid the use of any systemic or topical retinoids and antibiotics during the treatment course.

Table 1. Baseline characteristics of the patients with acne vulgaris and/or acne scars

\begin{tabular}{lccccc}
\hline \multirow{2}{*}{ Center } & \multicolumn{2}{c}{ Number of patients } & & \multicolumn{2}{c}{ Age (years) } \\
\cline { 2 - 3 } \cline { 5 - 6 } & Male & Female & & Range & Mean \\
\hline India & 76 & 43 & & $21-34$ & 29.5 \\
Saudi Arabia & 51 & 58 & & $20-33$ & 28.5 \\
Korea & 68 & 20 & & $18-28$ & 26 \\
Total & 195 & 121 & & $18-34$ & 28.2 \\
\hline
\end{tabular}




\section{Objective clinical assessment}

Photographs were obtained under identical camera settings, lighting, and patient positioning at baseline and at two months after the final treatment session. Objective clinical assessments were performed in a blinded fashion by two dermatologists who compared before and after photos using a global improvement scale Igrade 0 , worsened; grade 1,0-25\%, minimal improvement or steady state; grade 2, 26-50\%, moderate improvement; grade $3,51-75 \%$, marked improvement; and grade 4 , more than $75 \%$, near total improvement). The photographs were evaluated to evaluate improvements in inflammatory acne and acne scars. Investigators additionally assessed and recorded possible side effects, including bleeding, oozing, post-therapy dyschromia, scaling, crusting, folliculitis, worsening of inflammatory lesions, erythema, and scarring, at each follow-up visit at 1- to 2-week intervals. All recorded side effects were reassessed at two months after the final treatment.

\section{RESULTS}

At two months after the final treatment, grade 3 improvement was noted in 133 (42.1\%) of the 316 patients, followed by grade 4 improvement in 84 (26.6\%), grade 2 improvement in 78 (24.7\%), and grade 1 improvement in 21 (6.6\%) (Fig. 1). Objective clinical assessment using the global improvement scale revealed a median clinical improvement score for the 316 patients of $2.8 \pm 0.9$.

Fractional bipolar RF treatment using non-insulated microneedles was therapeutically effective against all types of acne scarring, including icepick, boxcar, and rolling (Fig. 2 and 3) ( $p>0.05)$. Distensible retractile and nondistensible scars exhibited remarkable clinical improvement; additional effects of improved skin texture and tone from the delivery of RF energy via the minimally invasive non-insulated microneedles were also observed. Inflammatory acne lesions showed improvements in both

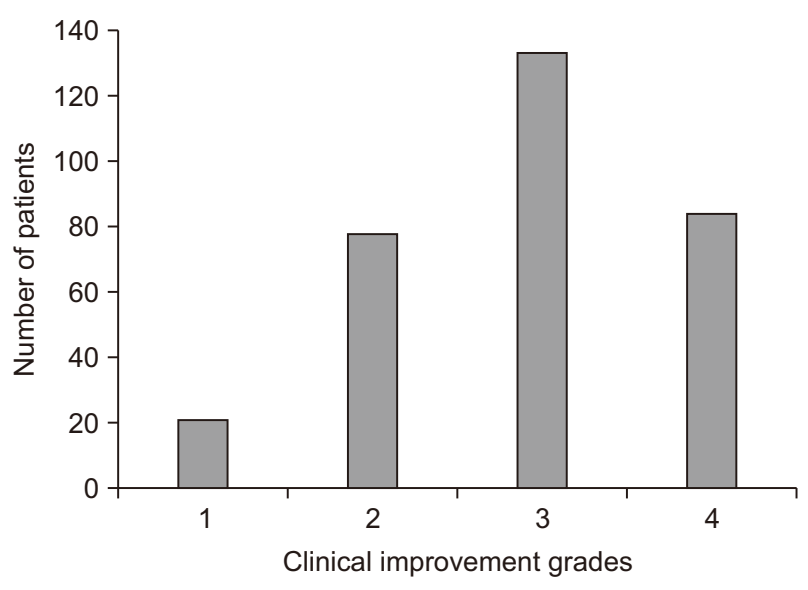

Fig. 1. Clinical improvement grades for the 316 patients treated with bipolar non-insulated microneedle RF treatment of acne vulgaris and/ or acne scarring.
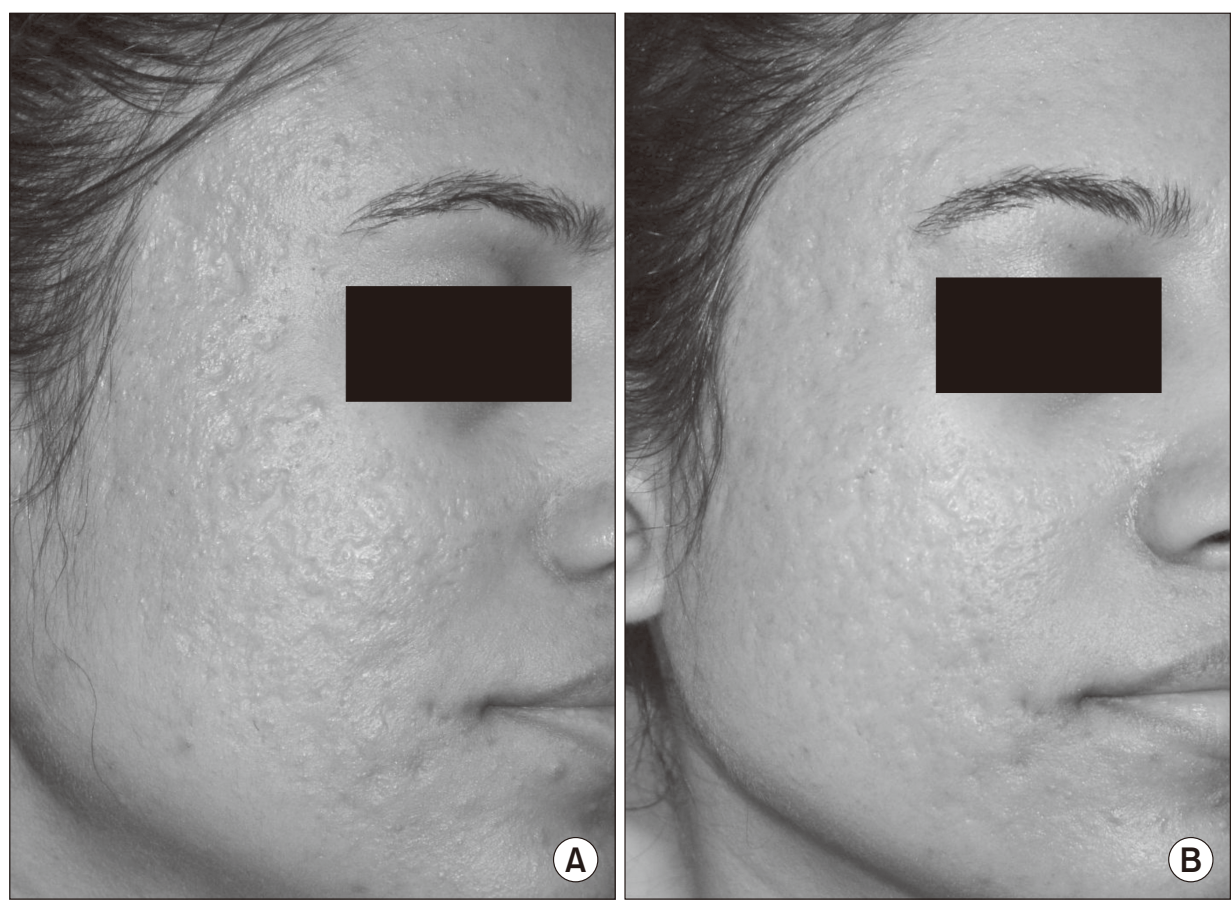

Fig. 2. Marked improvement of acne scarring in a 26-year-old female patient from India after two sessions of bipolar non-insulated microneedle RF treatment. Photos were taken at (A) baseline and (B) two months after the final treatment. 

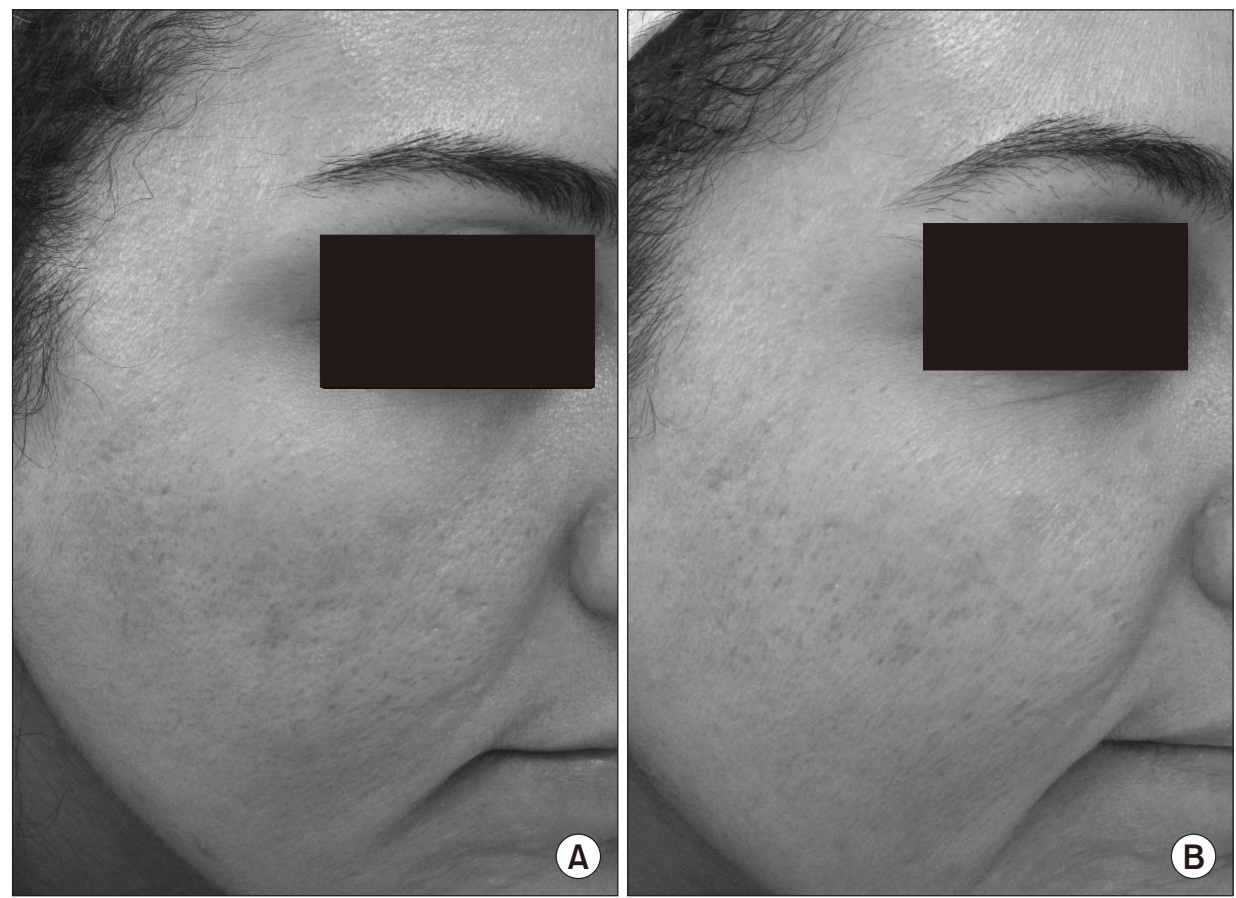

Fig. 3. Marked improvement of acne scarring in a 33-year-old female patient from Saudi Arabia after two sessions of bipolar non-insulated microneedle RF treatment. Photos were taken at (A) baseline and (B) two months after treatment.
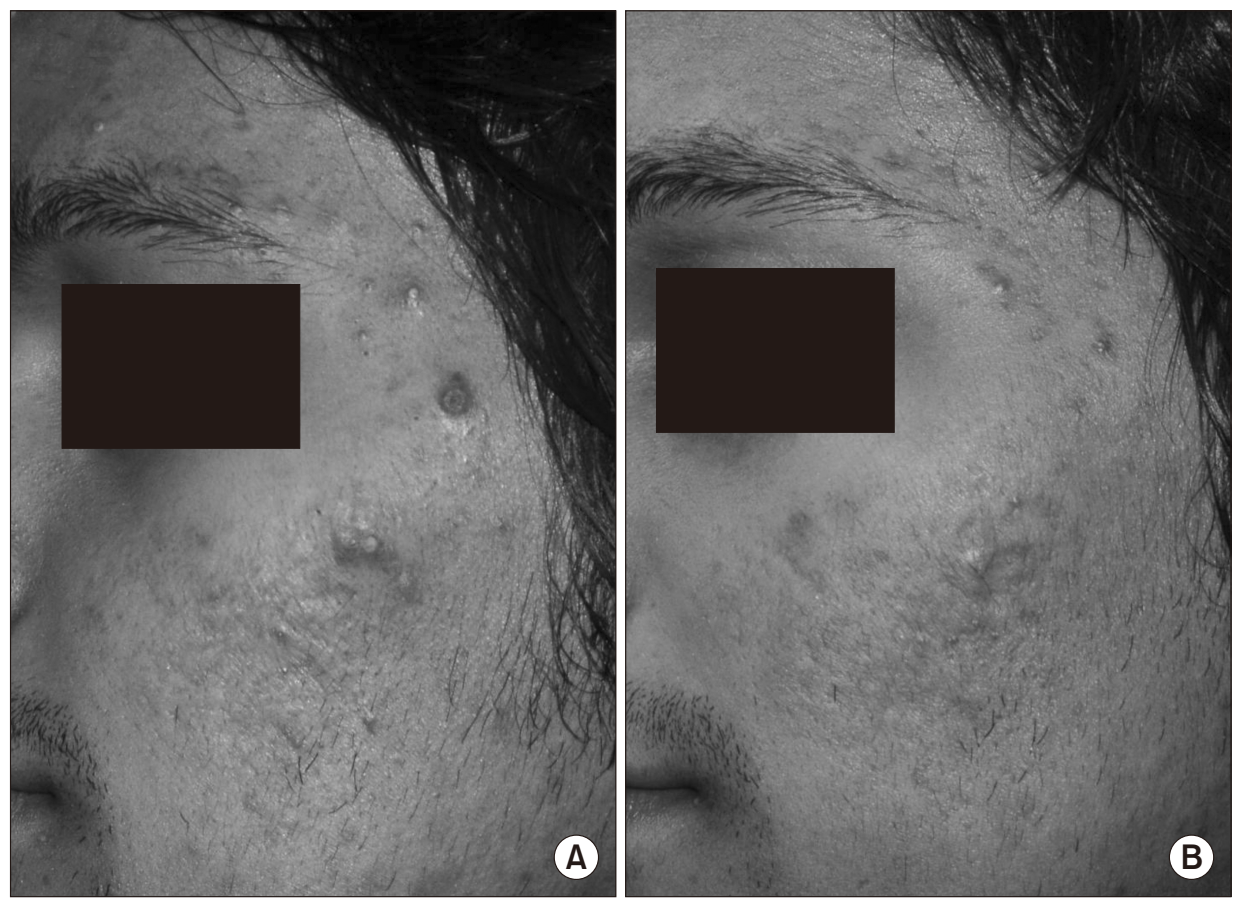

Fig. 4. Marked improvement of acne vulgaris and acne scarring in a 29-year-old male patient from India after two sessions of bipolar noninsulated microneedle RF treatment. Photos were taken at (A) baseline and (B) two months after treatment.

the number and severity of suppurative lesions (Fig. 3-5). Meanwhile, no significant differences in overall clinical improvement scores were recorded among the patients from India, Saudi Arabia, and Korea ( $p>0.05$ ).

Most of the patients well tolerated the pain incurred during treatment. Post-treatment bruising or purpura was not observed in any patient, even those with active inflammatory acne lesions. Throughout the course of RF treatment and two months after the final treatment, temporary aggravation of acne vulgaris or folliculitis was found in only 9 (2.8\%) of the 316 patients; all of the lesions spontaneously resolved within three weeks. Otherwise, no remarkable side effects were recorded. 


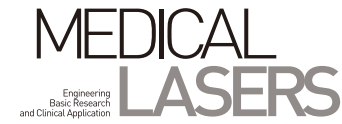

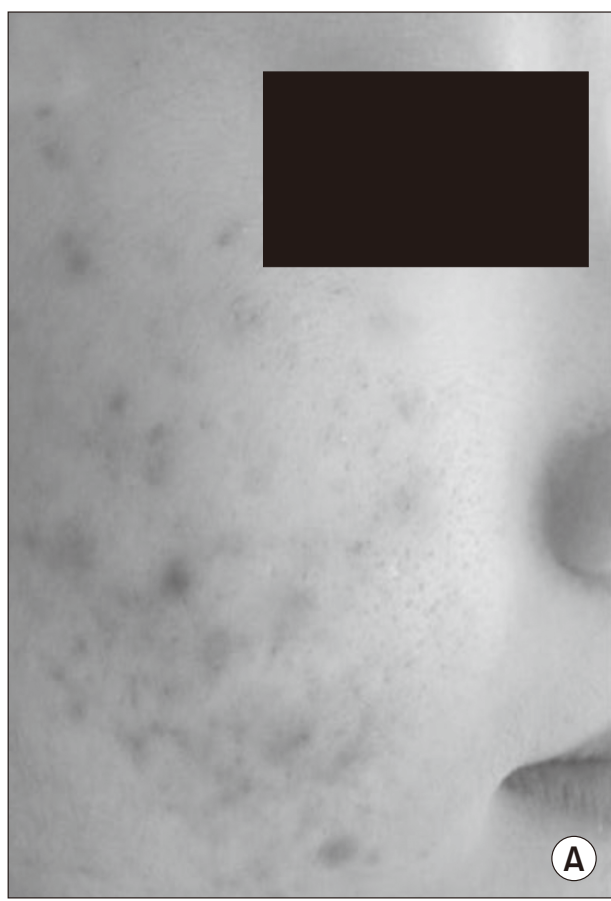

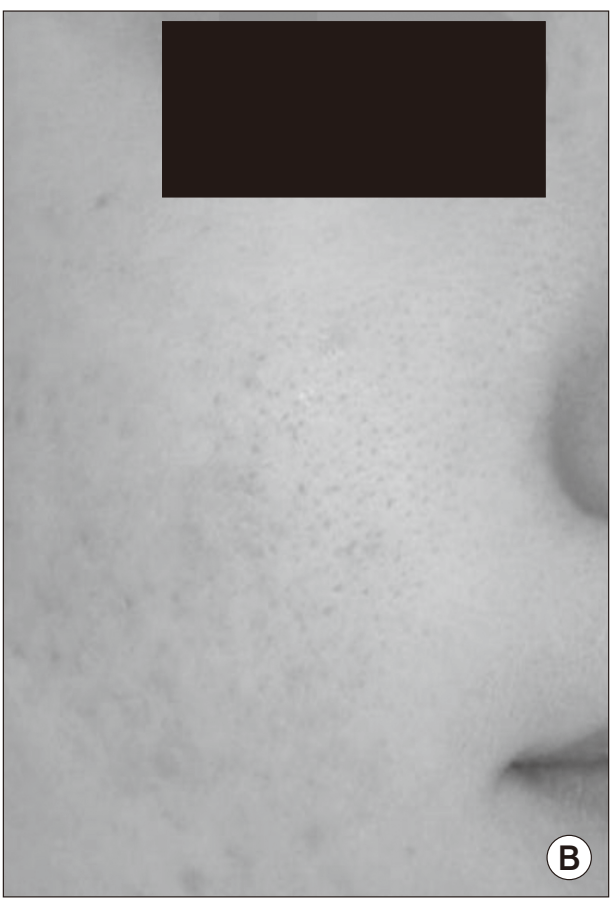

Fig. 5. Marked improvement of acne vulgaris in a 21-year-old female patient from Korea after two sessions of bipolar non-insulated microneedle RF treatment. Photos were taken at (A) baseline and (B) two months after treatment.

\section{DISCUSSION}

Several reports on microneedle RF systems and their effectiveness and safety in the treatment of acne vulgaris and acne scars can be found in the literature. ${ }^{11,13}$ However, few address non-insulated microneedle RF treatment in patients of Fitzpatrick skin types IV-V. Herein, via retrospective analysis of clinical photographs, we found bipolar non-insulated microneedle RF treatment to be safe and effective in treating inflammatory acne lesions and scars in patients of Fitzpatrick skin types IV-V from India, Saudi Arabia, and Korea. The majority of patients 168.7\%, 217 patients) showed either marked or near total improvement (grade 3 or 4 ) in their overall complexion, with no long-term side effects.

RF devices are frequently used for the treatment of atrophic scars, hypertrophic scars, and inflammatory acne. ${ }^{10,14}$ RF energy is absorbed by water, collagen, melanin, and dermal microvasculature, thereby producing a bulk heating effect on the dermis to induce cellular mediator and growth factor secretion, which results in wound healing. ${ }^{14}$ In the present study, the emission of bipolar RF energy via non-insulated microneedles immediately generated fractionated thermal edema on the treated skin. Thus, as with other RF devices, ${ }^{10}$ this heating effect most likely induced the inhibition of sebaceous gland activity and the promotion of wound healing processes, including neocollagenesis and the activation of growth factors.

The skin is composed of three major layers: the epidermis, dermis, and subcutaneous fat tissue, all of which show great differences in their tissue impedances. Our study suggested that the thermal response induced by the delivery of RF energy via non-insulated microneedles to the epidermis is weaker than that for the dermis, despite delivering the same electrical power to both layers. This resulted in a dermal heating effect that has proven to be effective in treating acne vulgaris and acne scar without emitting excess heat to the epidermis. ${ }^{10}$

Additionally, whereas the microneedles of a RF device used in a previous report were proximally insulated to protect the epidermis from RF heating at insertion sites, those used in the present study were not. ${ }^{15}$ Nevertheless, none of the side effects associated with RF heating on the epidermis, including burn, crusting, prolonged erythema, post-treatment dyschromia, and scarring, were observed. Instead, we observed clinical improvements in skin texture, skin tone, and enlarged facial pores. In comparison to insulated microneedle RF treatment, we suggest that non-insulated microneedle RF treatment may stimulate less of thermal response in the epidermis for visible improvements in skin texture and tone.

Our study demonstrated that non-insulated microneedle RF treatment is safe and effective in treating acne vulgaris and acne scars in patients of Fitzpatrick skin types IV-V, without epidermal complications, such as 
burning and scarring. Any pain incurred during treatment was well tolerated by most of the patients to whom topical anesthetic creams were applied, and all instances of post-therapy folliculitis spontaneously resolved with three weeks of the final treatment. We believe that our data could serve as a useful reference for designing future prospective studies, which will be needed to confirm our findings.

\section{ACKNOWLEDGMENTS}

We would like to thank Anthony Thomas Milliken, ELS, at Editing Synthase (https://editingsynthase.com) for his help with the editing of this manuscript.

\section{REFERENCES}

1. Harper JC. An update on the pathogenesis and management of acne vulgaris. J Am Acad Dermatol 2004;51(1 Suppl):S36-8.

2. Seaton ED, Charakida A, Mouser PE, Grace I, Clement RM, Chu AC. Pulsed-dye laser treatment for inflammatory acne vulgaris: randomised controlled trial. Lancet 2003;362:1347-52.

3. Munavalli GS, Weiss RA. Evidence for laser- and light-based treatment of acne vulgaris. Semin Cutan Med Surg 2008;27: 207-11.

4. Rinaldi F. Laser: a review. Clin Dermatol 2008;26:590-601.

5. Glaich AS, Friedman PM, Jih MH, Goldberg LH. Treatment of inflammatory facial acne vulgaris with combination 595-nm pulsed-dye laser with dynamic-cooling-device and 1,450-nm diode laser. Lasers Surg Med 2006;38:177-80.

6. Orringer JS, Kang S, Hamilton T, Schumacher W, Cho S, Hammerberg C, et al. Treatment of acne vulgaris with a pulsed dye laser: a randomized controlled trial. JAMA 2004;291:2834-9.
7. Cho SB, Jung JY, Ryu DJ, Lee SJ, Lee JH. Effects of ablative 10,600-nm carbon dioxide fractional laser therapy on suppurative diseases of the skin: a case series of 12 patients. Lasers Surg Med 2009;41:550-4.

8. Cho SB, Lee SJ, Kang JM, Kim YK, Chung WS, Oh SH. The efficacy and safety of 10,600-nm carbon dioxide fractional laser for acne scars in Asian patients. Dermatol Surg 2009:35:195561.

9. Lee SJ, Goo JW, Shin J, Chung WS, Kang JM, Kim YK, et al. Use of fractionated microneedle radiofrequency for the treatment of inflammatory acne vulgaris in 18 Korean patients. Dermatol Surg 2012;38:400-5.

10. Ruiz-Esparza J, Gomez JB. Nonablative radiofrequency for active acne vulgaris: the use of deep dermal heat in the treatment of moderate to severe active acne vulgaris (thermotherapy): a report of 22 patients. Dermatol Surg 2003;29:333-9.

11. Chandrashekar BS, Sriram R, Mysore R, Bhaskar S, Shetty A. Evaluation of microneedling fractional radiofrequency device for treatment of acne scars. J Cutan Aesthet Surg 2014;7:93-7.

12. Berube D, Renton B, Hantash BM. A predictive model of minimally invasive bipolar fractional radiofrequency skin treatment. Lasers Surg Med 2009;41:473-8.

13. Naouri M, Mazer JM. Non-insulated microneedle fractional radiofrequency for the treatment of scars and photoaging. $J$ Eur Acad Dermatol Venereol. In press 2015.

14. Elsaie ML, Choudhary S, Leiva A, Nouri K. Nonablative radiofrequency for skin rejuvenation. Dermatol Surg 2010;36: 577-89.

15. Hantash BM, Renton B, Berkowitz RL, Stridde BC, Newman J. Pilot clinical study of a novel minimally invasive bipolar microneedle radiofrequency device. Lasers Surg Med 2009;41: 87-95. 